\title{
Making metadata work in digital asset management and video game production
}

\begin{abstract}
John Horodyski
is the ESPN Integration Producer at Electronic Arts and is responsible for all integration of ESPN content with EA SPORTS titles. He works with the production teams at EA and ESPN in analyzing asset workflow and is responsible for aligning ESPN content integration with business partners from EA SPORTS titles at all EA studios. In his previous role as the World Wide Studios Digital Asset Manager, he was responsible for the identification and acquisition of assets for the studio digital asset management system and strategic research for DAM initiatives.
\end{abstract}

Keywords: metadata, media, video game

Abstract This article focuses on Digital Asset Management (DAM) and the metadata used to support and strengthen the asset to which it is related. A DAM solution involves both technical and human components, most notably the subjective understanding of the rich media assets being used and the creation and application of metadata for those assets. Making metadata work in DAM means investing in the construction of a metadata model, a container of descriptive elements about the assets, that enables the users to search for and retrieve the assets needed for their work. The production of video games will serve as the backdrop to this discussion; specifically, the unique digital assets used in a game's creation with some examples from Electronic Arts. Furthermore, this will provide the setting for an analysis of how DAM may be useful for the description, identification and retrieval of those digital assets so that they may be made accessible. Metadata must not be seen as a static indicator of knowledge, but rather as a fluid model that is always subject for review and for change in its ongoing support of the rich media digital assets in the DAM system.

Journal of Digital Asset Management (2006) 2, 255-261. doi:10.1057/palgrave.dam.3650041

Advances in technology have made it easier for the way in which information is accessed, distributed and ultimately reused in our day-today business operations. With these new advances, the interface between humans and computers is not only encouraged but also strengthened as a result of its permutation throughout all aspects of those operations. Information is no longer strictly associated with paper documents, with the use of rich media becoming more and more common. People want to be able to find, capture and reuse rich media assets in different contexts and for different purposes than originally intended. However, there are challenges associated with rich media; if the medium is the message, then using rich media forces the user to have a better understanding of those rich media assets needed for their business operations.

This paper focuses on digital asset management (DAM) and the metadata used to support and strengthen the asset to which it is related. A DAM solution involves both technical and human components, most notably the subjective understanding of the rich media assets being used and the creation and application of metadata for those assets. Making metadata work in DAM means investing in the construction of a metadata model, a container of descriptive elements about the assets, that enables the users to search for and retrieve the assets needed for their work. The production of video games will serve as the backdrop to this discussion; specifically the unique digital assets used in a game's creation with some examples from Electronic Arts. Furthermore, this will provide the setting for an analysis of how DAM may be useful for the description, identification and retrieval of those digital assets so that they may be made accessible.

According to a study carried out by the research firm GISTICS, "production employees 
can save an average of 36 hours per year by using a digital asset management system to organize their jobs and processes, and their employers can realize return on investment (ROI) of between eight and 14 times over a three-year period." "The more information there is to manage, the longer it will take to locate, manipulate and store that information, a process that should not need to be cumbersome to the user. As it has been argued that the basic tenet of Records Management is to provide the right information to the right person at the right time, in the right place, and in the right format, it may be understood that access to that information is a critical component to the process. Access may be strengthened by the descriptors put in place to describe ad define the assets in the DAM system: descriptors as found in a well-constructed metadata model.

There are as many variances of the term "digital asset" as there are misconceptions as to what the term actually entails:

Asset: an element of work produced by artists, musicians, game designers, or external contractors. This may include sound effects, movies, menu text, voice-overs and any other items that could be termed as "data" in the finished game. ${ }^{2}$

"asset ... something that has intrinsic value, whether because it can be sold or used to sell something else."3

Examples of rich media range from 3D art, 2D textures, animation files, video, music or sound. In fact, within each of these media are a variety of different formats for these media files. There is now a voluminous amount of media file types in existence and new file formats or newer versions of existing formats are released every year. Owing to the exponential growth of the computer software industry, careful consideration must be given to these existing formats and their new successors as to their long-term preservation. Furthermore, the ongoing addition of new formats is enough reason to be rigorous in the application of metadata to the assets so as to properly identify the assets for eventual retrieval and use. If a user is unable to use the asset because they do not have access to an application that is still compatible with the asset's (outdated) file format, then the DAM system has failed. ${ }^{4}$
The ability to define an asset with metadata may be the best way to provide any great level of detailed description for an item, description that may well aid in the retrieval of that item. Metadata is best defined as "data about data," and is needed in order to access the assets in the DAM system. The metadata is stored in a metadata model housing the many descriptive layers in association with the asset. It is difficult to apply descriptions of rich media digital assets due to the subjectivity of those applying the classification and description of the assets. Applying metadata allows for the description of an asset on different levels in the hopes that such a detailed description will assist in the retrieval and use of the asset. As more and more digital assets are used within a diverse range of industries, there is a need to define common metadata standards, an objective that many organizations are working towards. ${ }^{5}$ Yet, there are no specific standards directed at the video game industry, which is evidence of the nascence of this industry recognizing the value of the work created by designers, artists, and programmers. There is a unique "culture" to the work that is created in video game development and with that comes a multitude of independent methods, terms and understanding associated with the different game production methods. There is great potential to tap into this culture and attempt to create some standards and/or controlled vocabularies for video game production.

In order to apply metadata to digital assets used in the production of video games, reference to these other examples may provide the best guidance. DAM and its metadata considerations are not just a solution solely associated with the art department or graphic designers as it affects a much larger audience within an organization. The same digital asset that may be a critical asset to the marketing department may also be involved with the sales department, licensing as well as the art department. In fact, this is perhaps where a significant amount of potential confusion may rest, that is with those organizations not as familiar with the day-to-day management of rich media digital assets. And vice versa! The art department and graphic designers should also be expected to understand and know what type of descriptors the sales department, licensing, marketing, etc are using to 
help sell the product. An increased awareness and understanding of those rich media digital assets must be established in order to qualify the standards, resolution and file formats of the assets required for the video game in development, and for future versions under consideration (eg different languages for different markets and different technical platforms for the game consoles such as the Sony Playstation $3^{\mathrm{TM}}$ or the Microsoft Xbox 360 ${ }^{\mathrm{TM}}$ ). This awareness must not be made in isolation, but rather with a holistic view to the project with metadata construction as an integral component of DAM. Yet, at a micro-level, the specific source of rich media digital assets will also provide a unique insight into the origin of assets used in video game development. Assets may be available on a CD-ROM, as original photographs or prints, as copies in stock art collections (books) or as original digitally created assets with the aid of graphics or other design software. It is also useful to mention that the point of entry for many of the assets may be through a scanner, which adds another layer of problems for the implementation of a DAM system - digitizing the source in order to enter it into the system. It is arguable that the ingestion work associated with the implementation of a DAM system should not be underestimated in terms of resources to do the actual work.

The creation of a naming convention for assets and an authority file for those names is another consideration in metadata construction. Consider the dilemma of naming assets in order to achieve some leverage over version control. There may be an appearance of asset multiples (eg grasstexture.bmp, grasstexture_old.bmp, grasstexture_new.bmp) due to little relevance in the naming of these assets plus an artist may not know what other artists are working on, causing possible duplication and therefore redundancy in the productivity of the team (the complexities of this problem of file names will be explained later). Yet, if software can track the changes, then the intellectual exercise of securing a controlled vocabulary may then at least begin. DAM software is capable of automatically assigning names to items, thereby avoiding duplicate file names. If the convention is detailed enough, the system can do even more work by finding new files and automatically entering them into the system, if they follow the same naming convention.

This is definitely a contentious area because many people like to use "descriptive" names and will resist losing control over their file names. The DAM system can handle either case, but having a strong naming convention can make this management a lot easier. ${ }^{6}$ Yet, there is a level of skepticism with releasing the control of the intellectual management of assets to an automatic indexing system supplied by the software. For instance, if the assets were automatically assigned numerical dates, then how well would users be able to identify those assets? There may be several hundred assets accessed each day, yet there would be no descriptive identification for these assets other than a date. There may well be a low degree of usability in this situation if assets could only be located simply on the basis of a numerical date. Yet, there are even more challenging problems associated with the naming of files on a more descriptive basis. An image of a dog on a beach with its owner may mean many things to many people (eg beach landscape, animals, humans, happiness, etc). It is difficult to assume an understanding of what things may be "about" or "of" without knowing the context for each situation.

A structured vocabulary is the best example of a naming convention and ensures the consistency necessary for precise retrieval of information. Sources such as the Art \& Architecture Thesaurus, the Thesaurus of Geographic Names, the Union List of Artist Names, ICONCLASS and the Library of Congress Subject Headings provide a wide range of controlled terminology to describe the people, places, things, events and themes depicted by images. Some documentation refers specifically to surrogate images rather than to original works of art or artifacts. The Visual Resources Association (VRA) Data Standards Committee is developing a set of guidelines for the documentation of surrogate representations of original works of art and architecture. Unfortunately, there are no structured vocabularies specific to the nomenclature and jargon of the video game culture and the administrators of the DAM system should develop their own taxonomy or thesaurus of terms in order to maintain control over the 
assets. A structured vocabulary may well aid in the creation of a file naming convention for the organization as a whole and more importantly the creators of the assets themselves.

As a point of reference, the following fields and their respective definitions are examples of what types of metadata may be used for the description of digital assets in a video game.

1. Location Information - Information relevant to the location of the asset and/or its relevant copies (eg location of a digi-beta tape on a shelf in the library).

2. Creator - Person, persons or corporate entity responsible for the creation of the digital asset, including artists, graphics designers and stock-shot photograph companies (eg COREL, CORBIS).

3. Date Created - The date of creation of the asset (eg this may be the date of publication of an image on a disk (eg COREL 1998) or the date of creation of an image by an artist).

4. Asset Name (Record) - The name of the asset as given to it by the person(s) who created it or the name as applied by the individual responsible for uploading/ ingesting the asset (or cataloguer).

5. Asset Description (Notes) - Textual description of the digital asset that may be useful for searching and retrieving the assets, for example animals, Corinthian columns, Level 3 - Warehouse Location with dusk lighting, etc.

6. Categories - The subject category(ies) or classification applied to an image and/or image collection as applied. For example, the category of boats may include several catalogues of images of all the different types of boats.

7. Asset Reference - Refers to the path in which the asset resided on the computer (eg which network drive, folder, etc) before its ingestion into the DAM system.

8. File Format - The specific file format associated with the asset. Examples are JPEG, PSD, MOV, AVI and MPEG. It is important to note that some file formats (like AVI) can act as containers for different types of media and for media that is encoded with different codecs (ie DivX and $\mathrm{XviD}$ ).
9. File Data Size - Refers to the size of the data storage in the repository (or file system) per file (eg $43 \mathrm{MB}$ ).

10. Copyright - The legal rights that protect creative works from being reproduced, performed or disseminated by others without permission. The owner of copyright has the exclusive right to reproduce a protected work to prepare derivative works that only slightly change the protected work, to sell or lend copies of the protected work to the public, to perform protected works in public for profit, and to display copyrighted works publicly. These basic exclusive rights of copyright owners are subject to exceptions, depending on the type of work and the type of use made by others.

11. Team Project/SKU - Identifies the game team to which the asset belongs; SKU stands for Stock Keeping Unit and is a number associated with a product for inventory purposes (this will be a unique number identifying the asset for the game team).

12. Version Date - Refers to the version of the asset in the DAM system (eg December 2003, February 2005).

13. Thumbnail - Visual representation of the record with a miniature display of the catalogued asset in the display and/or record pane.

For any metadata model to be effective, there must be a significant level of metadata analysis, which should include user interviews in order to establish what information is needed by the users of that DAM system for them to search and retrieve assets. These interviews should be conducted with the creators of the assets as well as the users. It may well be shown that different teams may have different naming conventions as well as different descriptors, making the work all the more challenging. Yet, having both the creators and the users contribute to the description and creation of metadata for the asset will increase the amount of descriptive information available about the asset, which will ultimately assist in the search and retrieval for the asset.

There is much difficulty in describing an image with words because it is the ultimate 
exercise in subjectivity. There is a complete reliance on the user's judgment to classify assets, a process that is almost always done through the use of specific keywords. This process requires great dedication and care from the people in charge of classifying the information $(7 \mathrm{~min}$ average for each image, in the case of multimedia publishers, even without any subsequent revision). ${ }^{7}$ As the classification is produced mainly on a personal subjective manner, the obtained results are neither standard nor reliable. Furthermore, there is the fear that these classifications create artificial boundaries that may actually obscure important relationships (although the generous use of metadata may provide some relief here). It is arguable that algorithms may fare well in automatically indexing or assigning unique identifiers (eg numbers), but they do not have the ability to provide subject classification to images. Human interaction with the rich media digital assets becomes an integral part of metadata analysis when creating a DAM system.

As an example, graphic artists looking for "alien landscapes" may be more interested in searching for a particular shape or texture rather than a particular color. In addition to this more simplistic regimen, Vlad Weilbut argues that other users may prefer various levels of sophistication of respective interfaces: less demanding ones will be satisfied with a simple color wheel for selecting approximate hues in desired images, while domain experts may demand a wide range of controls enabling them to specify exact levels of saturation, lightness, etc. ${ }^{8}$ As long as textual identification is used to query a non-textual asset, this will continue to plague the retrieval of relevant items in a query. Creative methods to identify assets, however, may provide some relief and perhaps more relative returns to a query. In the future, it may be possible to retrieve images on the basis of visual criteria and current research into pattern recognition may prove fruitful, making it possible to retrieve images by colors or iconic shapes, or by the position of elements within the image frame. ${ }^{9}$ More often than not, there are ad hoc solutions to asset management problems ... solutions that suffer from confusion and frustration over file versions, file locations and the status of a digital asset that is integral to the project in operation. Consideration for determining access to assets using technology as well as metadata analysis is needed.

The following rich media digital assets show the complexities of defining the metadata to be used:

\section{D MODEL (Figure 1)}

There are inherent challenges to creating thumbnails of 3D models due to the nature of these assets to be manipulated and changed as commanded by animation file scripts (3D models are the objects, characters, vehicles, props, etc that serve as the primary objects in the game). Challenges facing 3D models within a digital asset management system may be:

- Do you leave the 3D model in a "standard" position that is then replicated for all 3D models in your DAM system?

- Do you leave the 3D model "raw" with the skeletal system showing ... does that have meaning for your users?

- Do you show the thumbnail with the 3D model and its textures on?

- Is the polygon count per 3D model important to your users and, if so, should that then become a unique piece of metadata that may be queried?

- What about any possible relationships with this $3 \mathrm{D}$ model?

- What are the textures that are associated with this?

- Are there any animation files associated with this?

- Where was this 3D model used?

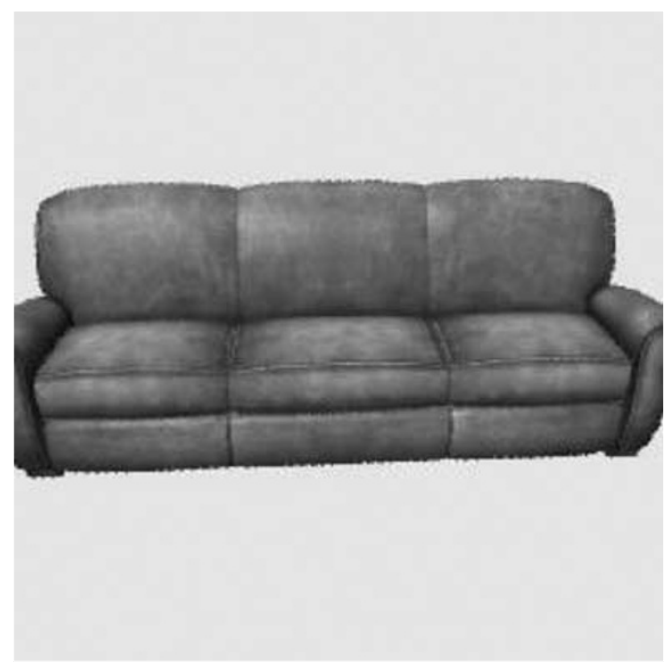

Figure 1: Sofa 3D model from the Sims 2 


\section{Textures (Figure 2)}

A texture is a digital file used to wrap or cover the skeletal structure of a 3D model to give it the color and appearance of a $3 \mathrm{D}$ object. The artists who create textures will know them well so this is a great opportunity to interview them and learn more about these assets that will be part of your DAM system. For instance, questions like:

- What file format was the texture created in?

- Will that texture be able to be used with a variety of $3 \mathrm{D}$ modeling software or is it best applied to a specific piece of software?

- Is it tileable?

- If the texture "looks" like grass, could it also be moss or dirt or better yet, what exactly is it?

- Is the asset the original high-resolution file needed to create further textures?

\section{Concept Art}

Concept art, a piece of original artwork either digital by origin or digitized from an original physical source, is often used as reference for artists during production and has some unique metadata considerations. For instance:

- Is the "subject" of the concept art based upon a real person or location and, if so, are there any licensing rights associated with that?

- Who created this work, when and is the digital asset the original or a copy?

- And if there is an original physical copy of the asset, where is that located?

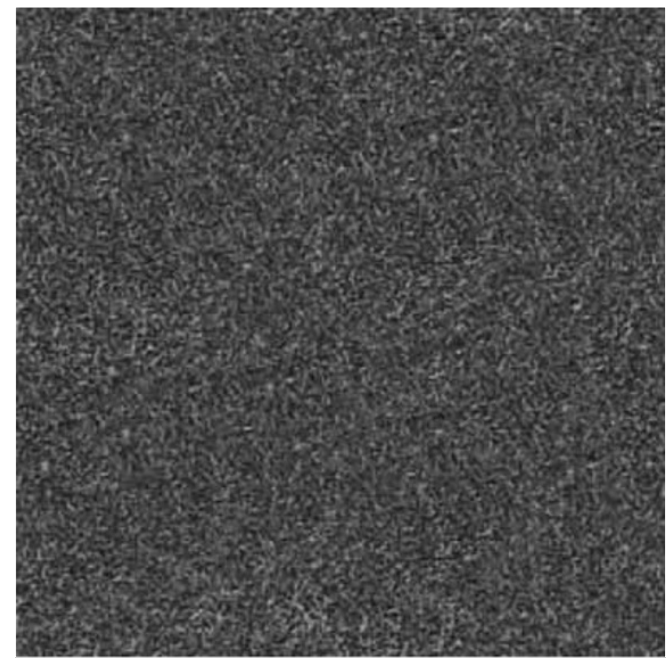

Figure 2: Grass texture from Need for Speed Underground
(Figure 3) Metadata model construction is more than just entering detailed metadata so that you can quickly track down the asset you need. It is taking the time to understand the digital asset and its unique qualities and then translating those into unique identifiers as created through metadata.

Here are some items to consider when creating metadata for rich media digital assets:

- Is long-term data preservation critical to the needs of the organization? Will we be re-using artwork from past projects and how will we be using them?

- Do your users need to be able to go back and look at old logo designs?

- Who are your users and how do they work?

- Are these assets iterative by nature?

- How do you achieve metadata consensus in a global setting?

- Are there any relationships between assets (ie specific logos for presentations)?

- Is your organization able to perform metadata capture at asset creation?

- Are your users satisfied with the visual presentation of the assets in the thumbnails?

- How well are you prepared for new asset types and their challenges?

Despite the considerations, resistance will occur at the level of asset ingestion and with cataloging and applying metadata to the assets. Indeed, it is time consuming and it may be understood as not that important or irrelevant to a DAM implementation. Yet, more time invested at the beginning of a DAM implementation with the digital assets will provide greater understanding of what those assets are, which in turn will produce richer metadata.

The management of rich media may be problematic for those desiring to find solutions

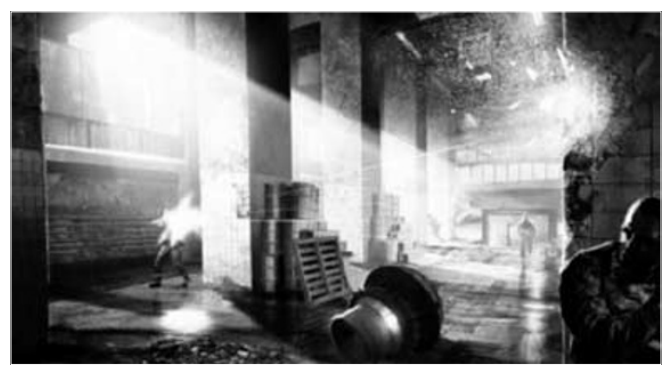

Figure 3: Concept art from BLACK 
based upon what has already worked in the past. This, however, is not to argue that traditional examples of information management have not been effective, rather that it affords new ways of thinking about how information is created, distributed, used and retrieved. There is a certain expectation that problems associated with technology must be solved with technology; it must be a part of the solution, but not the solution in itself. There must be a human component to the solution in order to support and complement the relationship between the new media (as applied through technology) and humans using rich media. The creation of a metadata model to identify and support rich media digital assets is a productive way in which to provide greater understanding of rich media assets. Yet, creating the metadata model does not constitute completion as the description of the assets is an ongoing process. From the original asset there is the potential for great reuse, which is made easier by better description and understanding of the asset for the organization. There is a direct need to invest time in documenting processes and in interviewing the "creative" person(s) responsible for asset creation and have them provide descriptions of those assets. In addition, anyone who uses the asset should also be interviewed because they will be searching for and retrieving the assets and may well use different descriptors than the creators. Metadata must not be seen as a static indicator of knowledge, but rather as a fluid model that is always subject for review and for change in its ongoing support of the rich media digital assets in the DAM system.

\section{References}

1 Hamilton, Alex 'Is Asset Management a Business?' Digital Publishing Magazine, (http://www. dpsmagazine.com/Features/F_2-2/assetmgmntAH. htm) (25 October, 2000).
2 Miller, Daniel 'Asset Management: Taming the Complexity', Game Developers Conference 1999 Proceedings, San Jose, CA, March 5-9: 701.

3 Hamilton: 1.

4 New occupations have been created out of necessity to assist in the management of digital assets. For example, "Data Wizards are inexpensive, less experienced team members who relieve the workload of more experienced and critical members of a game development team and let them focus on the core elements of the game. Data Wizards create and manage the content in a game throughout the use of game tools." While this position is industry specific (video game and entertainment industry), it is evidence that the volume of media and its ensuing problems within an organization is generating a role to be filled to solve that specific problem. More may be found in Taylor, Steven H. "Data Wizards and Wands", Game Developers Conference 2000, Proceedings, May 4-8, Long Beach, CA 263: 1.

5 Some of the groups and organizations include: The Metadata Coalition. (http://207.33.3.206/ welcome.html); and, the European Broadcasting Union (EBU) and SMPTE have formed a task force for the Harmonization of Standards for the Exchange of Program Material as Bit Streams. (http://www.smpte.org/engr/ebumeet1.html); as found in Rogozinksi, Beth. "Acquisition and Management of Digital Assets for the Transitioning Broadcast Facility." SMPTE Journal (March 1999): 159.

6 Duncan, Brian and Tylor Hagerman. 'Asset Tracking and Database Design', Game Developers Conference 1998, Proceedings, May 4-8, Long Beach, CA 161.

7 Encinas, Julio, Juan, Llorens and Antonio, Amescua 'Indexing and Classification of Images in Large Organisations', Libri 1999: 49: 16.

8 Weilbut, Vlad 'Computer, find me pink oval with some green dots! Image content searching is here', Berkeley Digital Library SunSite: Digitizing Images and Text, (15 October 2000). http://sunsite. Berkeley. EDU/Imaging/Databases/Fall95papers/ vlad2.html: 2.

9 Some of the newer search engines such as piXlogic or Riya are worth investigating. 